\title{
Learning teleoperation of an assistive humanoid platform by intact and upper-limb disabled users
}

\author{
Mathilde Connan, Marek Sierotowicz, Bernd Henze, Oliver Porges, \\ Alin Albu-Schäffer, Máximo A. Roa, Claudio Castellini
}

\begin{abstract}
With the advent of highly dexterous robotic arms, assistive platforms for home healthcare are gaining increasing attention from the research community. Control of the many degrees of freedom of such platforms, however, must be ensured uniformly, both for non-disabled and disabled users, in order to give them as much autonomy as possible. Nine users, including two upper-limb disabled, were challenged to complete highly complex bimanual tasks by teleoperating a humanoid robot with biosignals. The users were equipped with a light and wearable interface consisting of a body tracking device for guiding the torso and arms and two electromyography armbands for controlling the hands by means of interactive machine learning. All users were able to complete the required tasks, and learning curves are visible in completion time metric.
\end{abstract}

\section{INTRODUCTION}

$\mathrm{T}$ HE world around us is shaped to be operated by arms and hands, and the loss or impairment of the upper limb leads therefore to a dramatic degradation in the quality of living. A person with upper-limb amputation is prevented from swiftly acting in the world for the rest of her/his life, since state-of-the-art prosthetic or assistive solutions cannot usually operate more than one degree of motion, or if they can operate more than one, this can only happen sequentially, one motion at a time. Extensive application of statistical techniques to surface electromyography (sEMG) has revealed that, in controlled conditions, users can produce several discernible signal patterns corresponding to the intended actions to be performed by the absent limb. Unfortunately such techniques have so far shown little generalization power across users and when used online in daily-living environments, while, e.g., lifting weights and unpredictably changing one's body posture [1]. Attempts at solving this problem can be found, e.g., in [2], where unreliability is tackled using incremental machine learning (iML), i.e., an algorithm that can accommodate for new knowledge on the fly, in order to mend the instability of the intent detection system online. Degris and colleagues [3] as well as Nowak et al. [4] have explored the usage of reinforcement learning in the context of user/prosthesis interaction. Whether this idea works in practice, however, is still controversial [5].

This work was partially supported by the German Research Society projects Tact-Hand (DFG Sachbeihilfe CA-1389/1-1) and Deep-Hand (DFG Sachbeihilfe CA-1389/1-2). All authors are with the Institute of Robotics and Mechatronics, DLR - German Aerospace Center, 82234 Wessling, Germany. (Corresponding author: mathilde.connan@dlr.de)
In order to verify its effectiveness, we have designed an experiment in which both non-disabled and upper-limb disabled users were challenged to teleoperate a dexterous assistive humanoid platform using sEMG bracelets and a custom-made body posture detection device [6]. iML was employed to account for and correct instabilities of the intent detection system, whose model was updated whenever the user deemed the task to be unattainable. We hypothesized that such a setup and protocol would enable human users to complete all tasks, and that a learning effect would appear over time, leading, in the end, to uniform results across users, irrespective of their disability.

\section{MATERIALS AND METHODS}

\section{A. Experimental Setup}

Seven non-disabled users (28.4 \pm 7.1 yo) and two upperlimb disabled users (34yo, congenital absence of the right hand; 48yo, trans-radial bilateral traumatic amputation) were involved in this experiment. All subjects signed an informed consent form prior to the experiment.

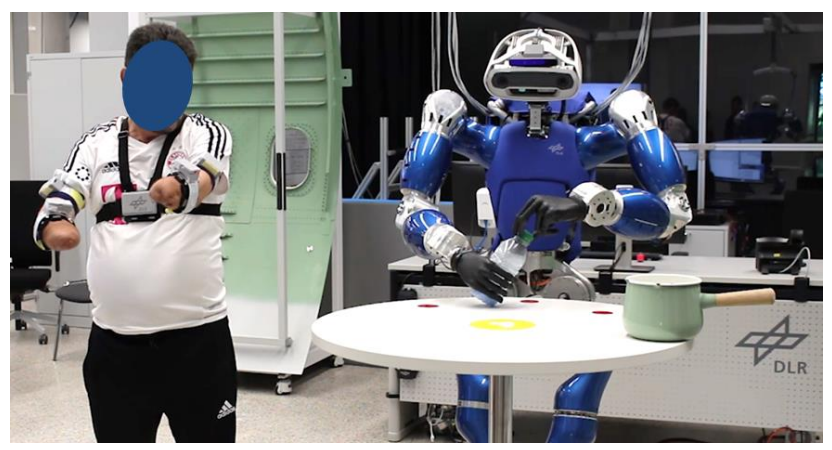

Fig. 1. A bilateral amputee performing highly complex bimanual tasks by teleoperating a humanoid robotic platform.

The users were equipped with a wearable upper-body tracking device (Fig. 1) based on inertial measurement units (IMUs) [6] placed on their forearms, upper-arms, and torso for controlling the position and orientation of the robot's hands, as well as, indirectly, its torso and arms thanks to the controller detailed in [7]. Additionally, a Myo armband from Thalmic Labs was placed on each of their forearms in order to record sEMG activity. From the sEMG signals, the desired hand poses of the robotic hands were predicted thanks to a ridge regressor with random Fourier features [8]. In the case of the impaired users, this regressor also predicted the wrist movements. For intact users, this was 
determined by the IMU-based tracking device with an IMU placed on the dorsal part of each hand. Due to the high instability resulting from the high number of hand and wrist poses to predict for the amputees, a slightly different training protocol was implemented for them in which only the taskspecific poses were trained by the machine learning algorithm. The humanoid platform TORO controlled by the users was developed at the German Aerospace Center (DLR) [7], [9].

\section{B. User Study Protocol}

The users were challenged to achieve highly complex tasks inspired from daily living activities and requiring a high level of bimanual coordination. The experiment consisted of three tasks, the first two being divided in two subtasks. The users had an unlimited number of trials and were free to stop or pause the experiment at any moment. Each task was performed four times. If an object dropped on the floor or if the experimenter judged that the user would not be able to recover a correct objects' setting, the task was reset to the initial setting of the subtask. The list of the tasks is visible in TABLE I. The subject performance was evaluated in terms of the time it took them to complete each task (Time to Complete Task, TCT).

TABLE I

DESCRIPTION OF THE TASKS

\begin{tabular}{cl}
\hline \hline Task ID & Summary of the task \\
\hline $1 \mathrm{a}$ & Take the lid off the pot and place it on the table \\
$1 \mathrm{~b}$ & Take the ball, put it in the pot, place back the lid \\
$2 \mathrm{a}$ & Unscrew the cap of the bottle \\
$2 \mathrm{~b}$ & Pour the bottle's content into the pot \\
3 & Press a sequence of buttons on the phone \\
\hline \hline
\end{tabular}

\section{RESULTS}

The summary results on the TCTs are given in Fig. 2. A decrease in the TCTs is visible when considering each task and repetition. The one-sided amputee (D1) achieved better results than the pool of subjects for the first three tasks. For the last two tasks his TCTs are higher but still comparable. The double-sided amputee (D2) also presents TCTs in line with the non-disabled users. The improvement ratio of the TCTs, between repetition 1 and 4, ranges from 3.9 times (Task 1a) to 1.2 times (Task 1b), with an average over all tasks of 2.2 times (1.49 for D1 and 2.41 for D2).

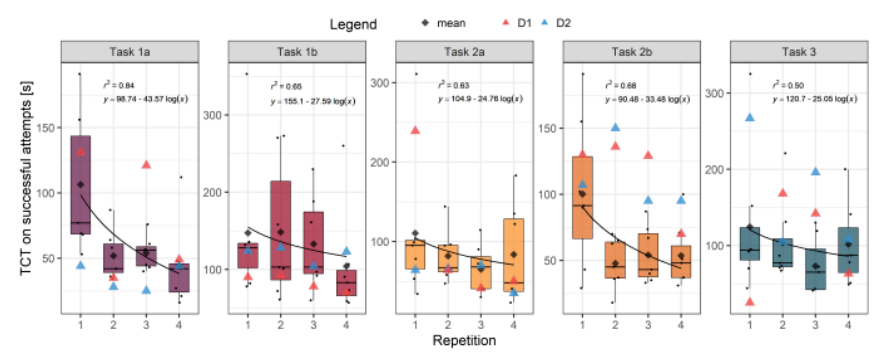

Fig. 2. Results of the user study on successful attempts.

\section{DISCUSSION}

We have presented here an assistive platform for dailyliving activities and put it to the test by challenging nondisabled users as well as users with different upper-limb disabilities to perform complex tasks requiring a high level of bimanual coordination. The experimental results confirm that, with the use of iML, all users were able to quickly and efficiently learn to teleoperate the platform and successfully complete all tasks, and that a learning effect was apparent, speeding up the execution of the tasks over time. Learning was uniform across seven non-disabled users and two upperlimb disabled persons, namely a person born with right-hand trans-radial congenital deficiency and a bilateral trans-radial traumatic amputated user. Previous research has also studied the use of accelerometry data in prosthetic control [10]. In future work, we intend to develop a machine learning algorithm fusing IMU and sEMG data in order to further improve the hand control without the necessity to train in several positions.

\section{References}

[1] A. Fougner, E. Scheme, A. D. C. Chan, K. Englehart, Ø. Stavdahl and O. Stavdahl, "Resolving the Limb Position Effect in Myoelectric Pattern Recognition," IEEE Transactions on Neural Systems and Rehabilitation Engineering, vol. 19, p. 644-651, 2011.

[2] J. M. Hahne, S. Dähne, H.-J. Hwang, K.-R. Müller and L. C. Parra, "Concurrent adaptation of human and machine improves simultaneous and proportional myoelectric control," IEEE Transactions on Neural Systems and Rehabilitation Engineering, vol. 23, p. 618-627, 2015.

[3] T. Degris, P. M. Pilarski and R. S. Sutton, "Model-free reinforcement learning with continuous action in practice," in IEEE American Control Conference (ACC), Montreal, QC, Canada, 2012.

[4] M. Nowak, C. Castellini and C. Massironi, "Applying radical constructivism to machine learning: A pilot study in assistive robotics.," Constructivist Foundations, vol. 13, 2018.

[5] C. Castellini, "Upper Limb Active Prosthetic systems-Overview," in Wearable Robotics, Elsevier, 2020, p. 365-376.

[6] M. Sierotowicz, M. Connan and C. Castellini, "Human-In-The-Loop Assessment of an Ultralight, Low-Cost Body Posture Tracking Device," Sensors, vol. 20, p. 890, 2020.

[7] B. Henze, M. A. Roa and C. Ott, "Passivity-based whole-body balancing for torque-controlled humanoid robots in multi-contact scenarios," The International Journal of Robotics Research, vol. 35, p. 1522-1543, 2016.

[8] A. Gijsberts, R. Bohra, D. Sierra González, A. Werner, M. Nowak, B. Caputo, M. A. Roa and C. Castellini, "Stable myoelectric control of a hand prosthesis using non-linear incremental learning," Frontiers in Neurorobotics, vol. 8, p. 1-15, 2014.

[9] C. Ott, M. A. Roa, F. Schmidt, W. Friedl, J. Englsberger, R. Burger, A. Werner, A. Dietrich, D. Leidner, B. Henze, O. Eiberger, A. Beyer, B. Bäuml, C. Borst and A. Albu-Schäffer, "Mechanisms and design of DLR humanoid robots," Humanoid Robotics: A Reference; Goswami, A., Vadakkepat, P., Eds, p. 1-26, 2016.

[10] A. Radmand, E. Scheme and K. Englehart, "On the suitability of integrating accelerometry data with electromyography signals for resolving the effect of changes in limb position during dynamic limb movement," JPO: Journal of Prosthetics and Orthotics, vol. 26, p. 185-193, 102014. 